\title{
Immune Effector Mechanisms of the Nitric Oxide Pathway in Malaria: Cytotoxicity versus Cytoprotection
}

\section{Hossein Nahrevanian}

Department of Parasitology, Pasteur Institute of Iran

\begin{abstract}
Nitric oxide (NO) is thought to be an important mediator and critical signaling molecule for malaria immunopathology; it is also a target for therapy and for vaccine. Inducible nitric oxide synthase (iNOS) is synthesized by a number of cell types under inflammatory conditions. The most relevant known triggers for its expression are endotoxins and cytokines. To date, there have been conflicting reports concerning the clinical significance of NO in malaria. Some researchers have proposed that NO contributes to the development of severe and complicated malaria, while others have argued that NO has a protective role. Infection with parasites resistant to the microbicidal action of NO may result in high levels of NO being generated, which could then damage the host, instead of controlling parasitemia. Consequently, the host-parasite interaction is a determining factor for whether the parasite is capable of stimulating NO production; the role of NO in resistance to malaria appears to be strain specific. It is known that NO and/or its related molecules are involved in malaria, but their involvement is not independent of other immune events. NO is an important, but possibly not an essential contributor to the control of acute-phase malaria infection. The protective immune responses against malaria parasite are multifactorial; however, they necessarily involve final effector molecules, including NO, iNOS and RNI.
\end{abstract}

Key Words: iNOS, malaria, nitric oxide, NO, RNI.

\section{Plasmodium: An ancient enemy in a new millennium}

Malaria is one of the commonest parasitic diseases affecting humans and animals in the world [1]. It is transmitted by infected mosquitoes, which inject sporozoites into the blood stream; these invade hepatocytes to start asexual reproduction, to form merozoites, trophozoites, and gametocytes [2,3]. Malaria remains a major threat to the well being of mankind in much the same way as it has since prehistory [4]. In spite of the numerous control strategies, including vector control, parasite treatment and environmental sanitation, there has been no reduction in the number of infected cases [3]. During the past few decades, it has become clear that malaria eradication in endemic areas is unlikely to be achieved by vector control and chemotherapy alone; therefore, more focus has been made on immunoprotective agents, especially vaccines [1]. Despite intensive research efforts, no vaccine against malaria that is both effective and suitable for mass production is yet available [5].

\section{Pathophysiology of malaria in the host}

The immune system of the host has a complicated reaction to malaria infection. Major immuno-pathological syndromes that occur include: cerebral malaria, hyper reactive malarial Received on 22 January 2006; revised 15 June 2006.

Address for correspondence: Dr. Hossein Nahrevanian, Department of Parasitology, Pasteur Institute of Iran, Pasteur Ave., Tehran 13164, Iran. E-mail: mobcghn@yahoo.co.uk, Phone / Fax: +98 2166969855.

The Brazilian Journal of Infectious Diseases 2006;10(4):283-292. (C) 2006 by The Brazilian Journal of Infectious Diseases and Contexto Publishing. All rights reserved. splenomegaly (HRMS), malaria nephropathy, pigment deposition, excessive anemia, white pulp hyperplasia, and many other physiological disturbances. Hypoglycaemia and hypotension are observed in severe human malaria [6-8]. Splenomegaly and hepatomegaly are frequent manifestations of acute malaria in both humans and rodents [9]. Jaundice, prolonged coagulation, failure of gluconeogenesis and sequestration of parasitized red blood cells (PRBC), indicate liver disfunction in malaria [10]. Anemia is an inevitable consequence of malaria; its degree corresponds to the duration and severity of the multifactorial infection [9].

\section{Immunity in malaria and host resistance}

Different effector mechanisms have different roles in immunity against malaria; these include antibody, mononuclear cell, cytokine and mediator activity. Two factors are mainly responsible for the ability of the host to resist malaria infection: immunological mechanisms and innate characteristics [1,3]. The parasite can escape from host immunity by antigenic variation, with periodic changes in the expression of its surface antigens, leading to non-protective immune reactions [7]. Antigenic material released by the parasite may act directly on B-cells and could lead to polyclonal activation. Both macrophages and non-specific Tsuppressor cells appear to be involved in immune depression, because they act as target cells for soluble factors released by the parasite [11]. T-cells are crucial during the erythrocytic stage of infection, and they produce immunoglubulins (IgG1, IgG2a, IgM, and IgE) [12,13], which activate macrophages and initiate a Th1 immune response, which are essential defenses in the early stages of malaria. Later there is an 
immunity switch to a Th2 response, with antibody-mediated mechanisms for the elimination of parasites [11]. Both Th1 and Th2 cells can protect the host against malaria infection. Th1 cells protect, in part, by means of the nitric oxide (NO) pathway, whereas Th2 cells protect by enhancing a specific IgG1 response [14]. The effector functions of macrophages include release of $\mathrm{H}_{2} \mathrm{O}_{2}$, reactive oxygen intermediates (ROI), reactive nitrogen intermediates (RNI), NO, TNF and by the production of at least 80 other cytokines and enzymes [15]. Macrophages can be stimulated by IFN- $\gamma$ and TNF- $\alpha$ to produce high levels of NO. They can kill erythrocytic stages of malaria parasites through various mechanisms, including phagocytosis of smaller parasites and secretion of many cytotoxic factors. Macrophages also act as killer cells by antibody-dependent cell-mediated cytotoxicity (ADCC) [16]. Two major Igs produced during malaria infection are IgG and IgM $[17,18]$. Possible effects of antibody action during parasite development include blockage of merozoite dispersion, inhibition of cell invasion, intracellular killing of erythrocytic stages, inhibition or reversal of cytoadherence and cooperation with various cells to increase killing by ADCC [7]. A large number of cytokines appear to be involved in malaria, i.e. TNF- $\alpha$, IFN- $\gamma$, GM-CSF, IL-1, IL-4, IL-6, IL-8, and IL-10 [7]. Moreover, to date, NO is known to affect the production of more than 20 cytokines, including IL-1, IL-6, IL-10, IL-12, IFN- $\gamma$, TNF- $\alpha$ and TGF- $\beta$, by various immune cells, including macrophages, T-cells, natural killer cells (NKC) and endothelial cells [19]. Consequently, it is suggested that multifactorial protection is responsible for immune responses in malaria infection [20].

\section{Nitric oxide; a friend or foe?}

There are contradictory reports about the role of NO and related molecules in malaria. Some researchers have proposed that $\mathrm{NO}$ is involved in the development of severe malaria, whereas others argue a protective role for NO [21]. Due to these contradictory evidences, it is still an open question as to whether NO is protective or damaging [22]. Although knowledge about the cytotoxic effects of NO is steadily increasing, we are still only beginning to understand how, why, when, and where cells are affected by NO. As we continue to understand how the immune system defends us against malaria infection, clinical trials of a new generation of vaccines and therapies are underway [23]. A recent hypothesis suggests that a vaccine for malaria could be aimed at neutralizing the effects of toxins rather than eliminating the parasite [24]. Consequently, the NO theory of malaria pathology may lead to novel ideas for vaccine, therapy and prevention.

\section{Detection of NO and its up and downstream molecules}

Since NO is associated with many different activities in the body, it is expected that its metabolism will change under a wide variety of conditions, leading to alterations in the concentrations of its metabolites [25]. NO is difficult to detect, because it is produced in small amounts under most conditions and has a short half-life [26]. Nitrite levels are usually at much lower concentrations than nitrate levels in body fluids; hence nitrate is the predominant ion. In addition, nitrite is difficult to measure reliably in samples, because it is rapidly oxidized to nitrate [25]. Therefore most of the NO produced in tissues by the three nitric oxide synthases (NOS) is eventually eliminated as nitrate, which is stable in body fluids [26]. Nitrite is a metabolically unstable product; it is a quantitative indicator of the highly unstable NO [27]. It has been suggested that RNI levels are equal to the amounts of both $\mathrm{NO}_{2}^{-}$and $\mathrm{NO}_{3}^{-}$ [28]. Consequently, under appropriate in vivo and in vitro conditions, accumulation of RNI would be a useful way to measure NO action [26].

Despite the importance of $\mathrm{NO}$ as a biological mediator, there are few methods for its detection. The most common assay uses the Griess reaction to measure nitrite; if it is applied after catalytic reduction of nitrates in samples, it measures both free nitrite and the larger nitrate pool. These values are commonly used as a measure of RNI. The Griess microassay (GMA) is less sensitive than electrochemical detection (ECD), but it can be applied to the low concentrations of relatively stable NO metabolites that accumulate both locally and away from the site of NO production. However, indirect determinations assaying NO metabolites as RNI could be misleading. Nitrite and nitrate may not only come from NO synthesis by the host; they can also come from the normal bacterial flora, parasites or the nitrate content of the diet [29-31]. The GMA, the mostfrequently-used method to measure stable RNI, has become popular because it can easily be applied in the laboratory without complicated equipment or expensive materials, even though it has limited sensitivity [31]. It is suggested that GMA registers RNI accumulating in the body, representing an early phase of NO involvement, whereas ECD measures recently-released NO. Direct NO detection and indirect measurement of RNI can give similar results [32], but this does not always happen [33]. Therefore, measurement of downstream, long-life stable RNI is more reliable rather the detection of short-living, unstable, NO, NOS and iNOS molecules [34].

Attempts to detect iNOS mRNA induction have given varied results, depending on the tissues sampled in the host and parasite and the time of sampling [35-37]. Some studies report iNOS mRNA induction in a variety of tissues and cells in hosts infected with various species of Plasmodia [20,21,3742]. Whereas every organ seems to be a mixture of cell types, including endothelial cells that produce eNOS, and macrophages that produce iNOS, high-output NOS activity can be due to both eNOS and iNOS production within a single cell [42]. iNOS induction in malaria and its involvement in the clearance of malarial parasites apparently varies according to 
the stage of infection, the degree of parasitemia, the strain of Plasmodia and the target organ or tissue being examined [34].

\section{NO and hemoglobin (Hb)}

Even if NO is cytotoxic for some species of Plasmodia, it would seem to be an unlikely effector molecule in vivo, as the parasite is constantly surrounded by $\mathrm{Hb}$, a very potent scavenger for this radical $[36,43]$. Hence, RNI accumulation cannot be detected in red blood cells (RBCs) [44]; the $\mathrm{Hb}$ prevents NO from reaching the parasite [36]. Oxy-Hb in RBC is a target for RNI, and nitrite reacts with this molecule to form either nitrosothiol-Hb or met-Hb $[45,46]$. Within tissues, NO rapidly binds with heme groups of enzymes, such as guanylyl cyclase [25], which subsequently, in the presence of oxygen and met-Hb reductase, rapidly converts met-Hb and nitrite back to $\mathrm{Hb}$ and nitrate [25]. During the erythrocytic stage of malaria, Plasmodium degrades $\mathrm{Hb}$ as a major source of amino acids for protein synthesis. This process includes the transport of $\mathrm{Hb}$ from the RBC cytoplasm to the parasite's acidic digestive vacuole [47]. Consequently, a possible antiparasitic action of NO and RNI against malaria is not well accepted. This is due to the belief that $\mathrm{Hb}$ universally scavenges $\mathrm{NO}$ and related molecules, and thus the RBCs act as a permanent sink for these molecules. It now appears that $\mathrm{Hb}$ involvement in NO scavenging is more complex. Under certain conditions in vivo, when RBCs are saturated with oxygen, $\mathrm{Hb}$ binds to NO; but when there is low oxygen tension, $\mathrm{Hb}$ readily releases NO [30]. The sensitivity of malaria parasites to NO increases with decreasing oxygen tension, because $\mathrm{Hb}$ binding of $\mathrm{NO}$ decreases at low oxygen tension, making NO available to exert an antiparasitic effect [48]. Hb may serve a dual role, as a scavenger, but also as a donor for NO. It is thought that an equilibrium between binding (NO scavenging) and unbinding (NO release) of $\mathrm{NO}$ to $\mathrm{Hb}$ is reached at a variable oxygen tension [30].

\section{NO and hemozoin (Hz)}

Parasitized red blood cells are phagocytosed by phagocytic cells, and accumulation of Hz-loaded macrophages is regularly observed in the spleen and liver during malaria. Hz appears to inhibit RNI and ROI production, and it decreases the overproduction of these mediators, causing malaria pathology [49]. Since soluble heme can damage biological membranes and inhibits a variety of enzymes in Plasmodia, $\mathrm{Hz}$ accumulation is necessary for parasite survival. NO could react with intra-parasitic molecules and participate in the incorporation of free heme into $\mathrm{Hz}$ [44].

\section{NO induction by bacterial LPS and malarial antigens}

Lipopolysaccharides (LPS) are believed to be potent inducers of macrophage ROI and RNI against a variety of intracellular and extracellular pathogens-[27]. They are also reported to be potent stimulators of NOS isoforms [50] and iNOS mRNA [51]. LPS usually synergizes with IFN-g for the expression of iNOS; but it can have antagonistic effects, depending on the concentration and sequence of the stimulation [19]. iNOS production is up-regulated in various cells by LPS, TNF- $\alpha$, IFN- $\gamma$, IL-1b and other immunological stimuli [52-54]. Optimal NO production by macrophages requires a primary signal by IFN- $\gamma$, plus a secondary signal, such as LPS. These macrophages are capable of producing large amounts of NO, following stimulation in vitro with small amounts of LPS [37]. Clinical similarities have been observed between bacterial endotoxins and malarial exotoxins [37], which suggests that the pathology of both might be mediated by soluble factors [55]. However, malaria parasites and LPS seem to act separately through different pathways; therefore, a combination of both pathways could have unpredictable consequences for the host. LPS are reported to be potent inducers of NOS proteins in vitro, but there have been different and conflicting reports about their effect, depending on cell line and dose [37,50,51,56-60].

\section{The role of NO in malaria: pathologic or protective}

Changes in NO levels may be pathogenic rather than protective in the malarial host. In nonlethal Plasmodia infections, early increases in NO could stimulate Th1 cell types to produce more mediators (RNI, ROI, $\mathrm{O}_{2}$ ) to control parasitemia during infection, which would be similar to natural immunization [61]. Late increases in NO production in the liver and spleen appear to have pathological consequences and have been associated with hepato-splenomegaly [62].

\section{Evidence of NO in knockout animals}

In literature reports, the involvement of NO and RNI in malaria, based on experimental approaches other than endogenous RNI assays, have been contradictory and inconclusive. Evidence from studies made with iNOS-deficient mice indicates that NO production is not a crucial factor for the development of murine cerebral malaria [63] or for resolving mouse Plasmodium infections [64]. However, it is possible that iNOS-deficient mice use alternative pathways to overcome in-born deficiencies; for example, there might be up-regulation of cNOS to compensate the deficiency of iNOS [64,65].

\section{Target organs in malaria for NO production}

Various studies highlight the important role of the spleen for the induction and maintenance of immunity to malaria infection. The spleen serves as a mechanical filter for the removal of PRBC and URBC $[6,66]$ by opsonization with antibodies and/or complements [10]. Although antibodies alone are not cytolytic, immune spleen cells and antibodies 
can interact to mediate cytotoxic reactions in malaria [67]. The killing of lethal malaria parasites probably occurs primarily in the spleen [2], and spleen-dependent resolution of acute malaria has been reported [68]. Host defense in primary malaria infection requires an intact spleen, not a sub-population of immune spleen cells [69]. Early during murine malaria infection, high iNOS mRNA coming from NO production in the spleen, but not in the liver, has a protective effect in the outcome of malaria infection [37]. The involvement of the spleen might be specific to the strain of Plasmodia [70]. Whether RNI has a key role in the splenic immune mechanism against Plasmodia, and whether overproduction is pathogenic or protective for the host, is not fully understood [71]. It is possible, therefore, that during infection with other Plasmodia, other tissues, in addition to the spleen, may also produce NO.

Some reports have focused on the role of other organs in NO interaction with malaria. Accumulation of PRBC by phagocytic macrophages is observed in both the spleen and the liver during malaria [49]. The liver as well as the spleen could play a protective role in murine malaria [37]. Primary induction of immunity against blood-stage parasites may occur in the spleen, although the liver may assume this function in the absence of the spleen [72]. NO is produced by various cells in the liver and it could serve as a defense against invading microorganisms and parasites [25]. RNI can prevent the development of the exo-erythrocytic stage in the liver [39].

\section{NO and cell dependency}

In the target organs, NO can be released from several cell sources, which contribute towards the protective immune response against intracellular parasites [36]. Histological changes in the spleen and liver with variations in populations of spleen macrophages have been reported in different hosts infected by the same Plasmodium [73], which demonstrates host specificity in cell immunity [74] and shows how Plasmodia strain can affect cell proliferation [75-77]. Malaria is associated with increased numbers of macrophages in the bone marrow, liver and spleen [1], with a major role for splenic macrophages in generating ROI and RNI, leading to intracellular malaria parasite damage [68]. While NO itself may not be inhibitory for parasite development, its downstream products, possibly generated by macrophages, do have anti-plasmodial activity [36].

Some reports suggest a role for other types of immune cells against malaria [78,79]. Some researchers found proliferation of $\mathrm{B}$ and T-cells in murine malaria $[79,80]$, especially against nonlethal strains [81]. Others have reported increased numbers of T-cells in murine [82] and human malaria [83]. Increases have also been reported in the total number of splenic $\mathrm{CD}^{+}$and $\mathrm{CD}^{+}$cells during malaria infection $[84,85]$ or upon resolution of infection [86].

Exo-antigens released from Plasmodia are able to stimulate macrophages to produce RNI [44]; toxic metabolites, including
$\mathrm{O}_{2}, \mathrm{NO}, \mathrm{H}_{2} \mathrm{O}_{2}$ [87] and Th1 cytokines (TNF-a, IL-12) or Th2 types (IL-1, IL-10) [88], act as an inducible antimicrobial mechanism. $\mathrm{NO}, \mathrm{O}_{2}$ and $\mathrm{ONOO}^{-}$may be derived either from Th1-cells or directly through stimulation of macrophages and polymorphonuclear (PMN) cells [89]. It is possible that other cell types, in addition to macrophages, produce $\mathrm{NO}$ in vivo in the spleen during malaria infection [37].

Although, it appears that spleen macrophages are activated to produce NO and cytokines during malaria infection, immunity to malaria is a complex process, hence the possible role of other immune cells in other tissues cannot be ignored. The effects of Plasmodium on the proliferation of immune cells and on their functioning have not been fully elucidated. Further research is needed to determine the exact role of macrophages in NO induction during malaria infection.

\section{NO variation in malaria: Plasmodium and host dependency}

The pathology and prognosis of malaria depends not only on the strain of Plasmodium, but also on the species and strain of the vertebrate host [90-92]. Differences in the pathologies of Plasmodia strains appear to depend more on the strain of the parasite rather than on the type of mouse, indicating that the nature of the parasite and/or its antigens are more important than the mouse strain in determining the host defense response [22]. The data revealed that RNI is correlated with the stage of disease and the degree of parasitemia. RNI appears to be unable to act against lethal Plasmodia, which might be because the parasite can release toxins to inhibit NO and/or RNI production. In contrast, RNI does appear to resolve non-lethal Plasmodia infections, which suggests that negative feedback by high levels of NO, in early and mid stages of infection, can reduce NO production in the late stages [93].

\section{NO modulation}

Compounds capable of increasing or decreasing NO concentration may have medical applications for a variety of disorders [7]. Most reports are on the use of NOS inhibitors to moderate NO production during malaria infection. The NOS inhibitor, $\mathrm{N}^{\mathrm{G}}$-monomethyl-L-arginine (L-NMMA) was found to have no antiparasitic effect on several malaria parasites in mice [36,94-96]. Another NOS inhibitor, aminoguanidine (AG) is also reported to have no effect against lethal [63] and nonlethal Plasmodia in mice [37,64,97]. This latter observation prompted Favre et al. [64] to suggest that the effect of NO production on resistance to malaria may be strain specific. Other researchers did not confirm the effects of NO [13,98], or of AG [40,64] and Nw-nitro L-arginine methylester (L-NAME) [99]. NO accumulation could have a protective role during infection, but this might be due to a reduction in pathology rather than antiparasitic effects [100]. Failure of NOS inhibitors to modify the progress of malaria in mice could result from a 
failure to adequately inhibit enzyme activity in target cells in vivo. However, it is possible that blocking NO synthesis with prototype inhibitors will harm the malarial host [9].

\section{NO- inducers}

Among the several NO inducers and donors, LPS gave the highest induction of NO. NO-induction by LPS was longer lasting than that provoked by S-nitrosoglutathione (SNOG), which may reflect slow release of LPS from an oily vehicle [101]. SNOG generates NO alone, whereas LPS produces other immune factors, including cytokines, such as TNF- $\alpha$, IFN- $\gamma$ and IL-1, along with NO. Primary stimulation by IFN-g can enhance this process to produce further production of IFN-g, TNF-a and NO, leading to limitation of the parasitemia [4]. It is hypothesized that application of NO donors is appropriate for a fast/short induction of NO in both in vivo and in vitro applications, but for long-term induction during malaria, several injections are required. The effects of stress due to multiple injections cannot be ignored; therefore, the addition of drugs to drinking water may be a preferable alternative strategy [101]. The time course and level of NO release by NO donors is suggested to differ between in vivo and in vitro applications [100]. This might be because other host mechanisms interfere and interact with the NO pathway to remove metabolites. Investigation of NO production by S-nitroso-Nacetylpenicillamine (SNAP) in vitro showed that although release of the molecule occurs over $24 \mathrm{~h}$, half the production happens during the first 4-5 h [100]. Stimulation with LPS in vitro is able to induce the production of high levels of $\mathrm{NO}_{2}^{-}$, but optimal NO production by murine macrophages requires a primary signal by IFN- $\gamma$, plus a second signal, such as LPS [37]. In addition to LPS, other substances have been reported to increase NO metabolites in activated macrophages, or to induce iNOS mRNA, including in vitro addition of ascorbate with combined LPS-IFN [59].

Administration of LPS could be considered as an antimalarial trial, because of the resulting sharp reduction of parasitemia and increasing survival rate. This therapeutic concept could be improved to design a novel antiplasmodial agent, with modulating of selective cell mediators; however, there may be concerns about using bacterial endotoxins in therapy [101].

\section{NO- donors}

There are some negative reports on the use of NO donors for the reduction of malaria pathology. They appeared not to limit this disease, e.g. the effects of Deta NONOate (NOC18) on both murine [99] and human malaria infections [47], SNAP on P. falciparum [102] and SNOG on $P$. berghei and $P$. chabaudi [100]. However, there is support for the role of NO donors in the elimination of Plasmodia, using SNAP or SNOG $[47,100]$.

\section{NO pathway: Th1 or Th2}

Protective immunity induced by repeated malaria challenges can involve either Th1 or Th2, depending on the genetic background of the host [103]. Th1 cells produce NO, IL-2 and IFN- $\gamma$, which activate macrophages to produce further NO. When the local concentration of NO reaches a certain threshold level, macrophages prevent further Th1 cell proliferation by feedback inhibition of IL-2 synthesis [14]. It is well documented, that Th1 cytokines activate macrophages, whereas Th2 cytokines down-regulate macrophage activity [37]. Th1 cells subsequently activate the NO pathway, to control a rapidly escalating acute parasitemia. Later, as the production of Th2 cells increases, IgG1-mediated immunity resolves patent infection [4]. It is suggested that Th1 cells play a role in protective immunity, either by directly producing NO [64], or by activating NO production by macrophages [104]. It has been proposed that in murine malaria infection, Th1initially predominates over a Th2 response, and that later Th2 predominates over Th1 [105]. Moreover, a Th1 response in the spleen early during malaria infection leads to macrophage activation and NO production in resistant mice, whereas a Th2 response appears to suppress NO production by splenic macrophages and results in severe and lethal disease in susceptible mice [37]. NO involvement in murine malaria appears to be a Th1-type event, which is reduced during a Th2-type response. This could explain why Th1 cells stimulated early in infection activate macrophages and PMN cells to produce NO and other factors, whereas later in infection, Th2-type cells promote antibody production by Bcells $[83,84]$. It is now believed that the Th1-Th2 decision is crucial for effective immunity during malaria; therefore, it may be advantageous for pathogens to subvert this decision [88].

\section{NO and cytokines; a combination}

Phagocytosis by granulocytes and macrophages, and production of cytokines in response to parasite molecules, leads to macrophage activation and production of ROI and NO, and activation of T-cells and NKCs, and production of IFN-g [83], which subsequently activates iNOS [2]. IFN- $\gamma$ plays an essential role in the early immune mechanisms induced by sporozoites, leading to parasite elimination [20], enhancement of inflammatory cytokines [106] and regulation of TNF- $\alpha$ and NO production [107]. It is also proposed that IFN- $\gamma$ plays a vital role in activating effector cells, such as macrophages, which may exert an anti-parasitic effect by the release of parasite inhibitory factors, such as TNF- $\alpha, \mathrm{H}_{2} \mathrm{O}_{2}$, and $\mathrm{O}_{2}$ radicals other than NO [94]. TNF- $\alpha$ is involved in the development of splenomegaly [108], by inhibiting the development of the liver stage of Plasmodia [20,27]. Although blocking of TNF- $\alpha$ reduces NO production, the antiparasitic effect of TNF- $\alpha$ does not work through NO [95]. A combination of IFN- $\gamma$ and TNF- $\alpha$ induces high amounts of NO, controlling 
the peak level of parasitemia [108], whereas co-treatment with IFN- $\gamma$, TNF- $\alpha$, IL- 1 and LPS exhibits a strong synergy for the expression of iNOS in mouse macrophages [109], and in rat [110] and human hepatocytes [54]. Endotoxins and cytokines act synergically to up-regulate gene expression for iNOS [58], whereas glucocorticoids down-regulate the iNOS mRNA [110]. The balance in the cytokine network is important for protective immunity. This balance includes the amount of cytokine released, the rate, the time and the site of production [111].

\section{NO regulation in malaria}

The classical view of iNOS as an enzyme that generates NO in the immune system can be attributed to the observation that small quantities of iNOS-derived NO are critical for signal transduction events during infection [112]. NO levels appear to be controlled by NO itself, as well as by external factors [113]. NO may be a self-regulatory molecule [14], and excessive NO, regardless of the source, can further downregulate NOS activity [113]. TNF- $\alpha$, either alone or in combination with IFN- $\gamma$, upregulates NO synthesis in vivo during the blood stage of malaria [108]. The presence of the various isoforms of NOS appears to be an important manifestation of NO regulatory processes. Low levels of NO, as synthesized by cNOS (eNOS, nNOS), are involved in physiological events, whereas high levels of NO, as produced by iNOS have a role in pathological consequences [113]. A novel negative feedback mechanism involving NO downregulation of iNOS gene expression has been reported; this may limit overproduction of NO, when its concentration is saturated in the circulation in vivo. The mechanism could be the stopping or reverse oxidation of arginine to citrulline $[14,97]$. NO may regulate other immune cells, e.g. maturation of NKC [112], suppression of leukotreine (LT) and cyclooxygenase 2 synthesis [114]. Detailed mechanisms of the signaling pathways and the transcription factors involved in the induction and expression of NOS are at present unknown [115]. However, the understanding of these molecular interactions and the regulatory mechanism(s) exerted by NO on cytokine synthesis, enabling the application of immunoregulatory mediators, would be of considerable therapeutic value for the treatment of some major diseases, including those involving infection, inflammation and autoimmunity [14].

\section{NO and gene polymorphisms; a novel aspect}

Genetic susceptibility to severe malaria is heterogeneous, complex and involves several genes. There are associations of certain gene polymorphisms in different populations with different malaria strains and epidemiologies [116]. All parasitic infections are affected by the genetic makeup of their hosts, and there is evidence that underlying genetic factors determine the virulence of Plasmodia [91]. The role of NO in protection in mice appears to be dependent on the host genetic background [13]. Furthermore, it is now believed that there is a relationship between polymorphism of the gene encoding NO synthase and disease severity [117]. Moreover, iNOS promoter mutations mediate antimalarial resistance in areas endemic for malaria [118]. This may indicate differences in the genetic profiles of each population or strains of malaria and the epidemiology of disease in these endemic areas [116].

\section{Host-parasite combination; NO dependency}

The results of studies on human malaria remain conflicting, and in rodent malaria the principle protector mechanisms vary between different host-parasite combinations [100]. Therefore, the importance of NO for the killing of malaria parasites may also differ between mice and humans [64]. These differences may be attributable to such factors as the tissue sampled, target organs, days since infection, degree of parasitemia, assay method, host species and Plasmodia strain. The presence of parasites resistant to the microbicidal action of NO may result in high levels of NO being generated, which could then play a role in host pathology rather than controlling parasitemia $[9,97]$. Consequently, the hostparasite combination will be a determining factor as to whether the parasite is capable of stimulating NO production [100] or whether NO in resistance to malaria appears is strain specific [64].

\section{Conclusions}

Taken together, the data provided by researchers highlight the fact that $\mathrm{NO}$ and/or its related molecules are involved in malaria; but the involvement is not independent of other immune events. It is clear that NO is an important, but possibly not essential contributor to the control of acute phase malaria infection. Although, the protective immune responses against the malaria parasite are multifactorial, and the final effector molecules that mediate parasite death are not known, NOS, NO and RNI clearly appear to be implicated.

NO is only part of an immunopathological complex against malaria infection, and the anti-parasitic function against Plasmodia does not involve only NO activity; a combination of $\mathrm{NO}$ and other immune factors is required to resolve Plasmodia infection. Selective delivery of NO synthesis inhibitors and promoters to malarial host tissues is also indicated as a potential novel therapy to inhibit this parasite or to prevent pathological symptoms. Possibly, NO comes from several cellular sources, which can contribute towards protective immune responses against intracellular Plasmodia. Further investigation defining these sources will be important for the understanding of cell-mediated defense mechanism(s) in malaria. 


\section{References}

1. Wernsdorfer W.H., McGregor S.J. Malaria, principles and practice of malariology. UK Churchill Livingstone Ltd, Vol. 2, 1988.

2. Good M.F., Doolan D.L. Immune effector mechanisms in malaria. Curr Opin Immunol 1999;11: 412-9.

3. Wakelin D. Intracellular Protozoa; survival within cells. In: Wakelin D. ed. Immunity to parasites, How animals control parasite infections. London, UK, Edward Arnold, 1988.

4. Taylor-Robinson A.W., Smith E.C. A role for cytokines in potentiation of malaria vaccines through immunological modulation of blood stage infection. Immunol Rev 1999; 171:105-23.

5. Taylor-Robinson A.W. Parasite variation provides hope for malaria vaccine design. Trends Microbiol 2001;9:157.

6. Garnham, P.C.C. Rodent species of malaria parasites, P. berghei, P. vinckei and P. chabaudi. In: Garnham, P.C.C. ed. Malaria parasites and other haemosporidia. Blackwell scientific publications, 1966.

7. Hommel M. Immunology of malaria. In: World Health Organisation. ed. WHO, Health co-operation papers, Quaderni di cooperazion sanitaria, 1996.

8. Hermsen C.C., Van de Wiel T., Mommers E., et al. Depletion of CD4+ or CD8+ T-cells prevents Plasmodium berghei induced cerebral malaria in end-stage disease. Parasitol 1997;114:7-12.

9. White N.J. Malaria Pathophysiology. In: Sherman IW. ed. Malaria, Parasite biology, Pathogenesis and Protection. 1st edition, ASM Press, Washington D.C., American Society for Microbiology, 1998.

10.White N.J., Ho M. The pathophysiology of malaria. In: White N.J., Ho M. ed. Advances in parasitology, Vol. 31, Academic Press Limited, 1992.

11.Collier L., Balows A., Sussman M. Parasitology. In: Collier L., Balows A., Sussman M. eds. Toply and Wilson's Microbiology and microbial infection., London, UK, 9th edition. Vol. 5, Toply and Wilson, 1998.

12.Phillips R.S., Brannan L.R., Balmer P., et al. Antigenic variation during malaria infection: the contribution from the murine parasite Plasmodium chabaudi. Parasite Immunol 1997; 19:427-34.

13.Taylor-Robinson A.W. Regulation of immunity to malaria: valuable lessons learned from murine models. Parasitol Today 1995;2:324-32.

14.Taylor-Robinson A.W. Counter-regulation of T helper 1 cell proliferation by nitric oxide and interleukin-2. Biochem Biophys Res Commun 1997;233:14-9.

15.Clark I.A., Al-Yaman F.M., Cowden W.B., et al. Does malarial tolerance, through nitric oxide, explain the low incidence of autoimmune disease in tropical Africa? Lancet 1996;348:1492-4.

16.Roitt I.M., Brostoff J., Male D. Immunology. London, 5th edition Mosby Publication, 1998.

17.Deans J.A., Cohen S. Immunology of malaria. Annu Rev Microbiol 1983;37:25-49.

18.Marsh K. Immunology of human malaria. In: Gilles H.M., Warrell D.A. eds. Bruce-Chwatt's Essential Malariology, London, UK, Edward Arnold. 3rd edition ed., 1993.

19.Bogdan C., Rollinghoff M., Diefenbach A. Reactive oxygen and reactive nitrogen intermediates in innate and specific immunity. Curr Opin Immunol 2000;12:64-76.
20.Tsuji M., Miyahira Y., Nussenzweig R.S., et al. Development of antimalaria immunity in mice lacking IFN-gamma receptor. J Immunol 1995;54:5338-44.

21.Chiwakata C.B., Hemmer C.J., Dietrich M. High levels of inducible nitric oxide synthase mRNA are associated with increased monocyte counts in blood and have a beneficial role in Plasmodium falciparum malaria. Infect Immun 2000;68:394-9.

22.Nahrevanian H., Dascombe M.J. The role of nitric oxide and its up/downstream molecules in malaria: cytotoxic or preventive? SE Asian JTrop Med Public Health 2003;34(S2): 44-50.

23.Modlin R., Rickinson A. Immunity to Infection. Curr Opin Immunol 2000;12:387-9.

24.Playfair J.H., Taverne J., Bate C.A., et al. The malaria vaccine: anti-parasite or anti-disease? Immunol Today 1990;11:25-7.

25.Ellis G., Adatia I., Yazdanpanah M., et al. Nitrite and nitrate analyses: a clinical biochemistry perspective. Clin Biochem 1998;31:195-220.

26.Granger D.L., Hibbs J.B. High-output nitric oxide: weapon against infection? Trends Microbiol 1996;4:46-7.

27.Mellouk S., Green S.J., Nacy C.A., et al. IFN-gamma inhibits development of Plasmodium berghei exoerythrocytic stages in hepatocytes by an L-arginine-dependent effector mechanism. J Immunol 1991;146:3971-6.

28.Sam H., Stevenson M.M. In vivo IL-12 production and IL-12 receptors beta1 and beta2 mRNA expression in the spleen are differentially up-regulated in resistant B6 and susceptible A/J mice during early blood-stage Plasmodium chabaudi AS malaria. J Immunol 1999;162:1582-9.

29.Anstey N.M., Hassanali M.Y., Mwaikambo E.D., et al. Nitric oxide appears protective in Tanzanian children with Malaria: Evidence for increased NO production in subclinical infection and suppressed production in clinical and cerebral malaria. In: Moncada S., Stamler J., Gross S., et al. eds. The biology of nitric oxide, London, UK, Portland Press, 1996.

30.Taylor-Robinson A.W. Nitric oxide can be released as well as scavenged by haemoglobin: relevance to its antimalarial activity. Parasite Immunol 1998;20:49-50.

31.Marzinzig M., Nussler A.K., Stadler J., et al. Improved methods to measure end products of nitric oxide in biological fluids: nitrite, nitrate, and S-nitrosothiols. Nitric Oxide: Biology and Chemistry 1997;1:177-189.

32.Rysz J., Luciak M., Kedziora J., et al. Nitric oxide release in the peripheral blood during hemodialysis. Kidney Int 1997;51:294-300.

33.Allen B.W., Piantadosi C.A., Coury L.A.J. Electrode materials for nitric oxide detection. Nitric oxide 2000;4:75-84.

34.Nahrevanian H., Dascombe M.J. Reactive nitrogen intermediate (RNI) levels inside and outside Plasmodium infected red blood cells in murine malaria. J Trop Med Parasitol 2003;26(1):13-9.

35.Tsutsui N., Kamiyama T. Transforming growth factor betainduced failure of resistance to infection with blood-stage Plasmodium chabaudi in mice. Infect Immun 1999;67:2306-11.

36.Jones I.W., Thomsen L.L., Knowles R., et al. Nitric oxide synthase activity in malaria-infected mice. Parasite Immunol 1996;18:535-8.

37.Jacobs P., Radzioch D., Stevenson M.M. Nitric oxide expression in the spleen, but not in the liver, correlates with resistance to blood-stage malaria in mice. J Immunol 1995;155:5306-13. 
38.Klotz F.W., Scheller L.F., Seguin M.C., et al. Co-localization of inducible-nitric oxide synthase and Plasmodium berghei in hepatocytes from rats immunized with irradiated sporozoites. J Immunol 1995;154:3391-5.

39.Seguin M.C., Klotz F.W., Schneider I., et al. Induction of nitric oxide synthase protects against malaria in mice exposed to irradiated Plasmodium berghei infected mosquitoes: involvement of interferon gamma and $\mathrm{CD}^{+} \mathrm{T}$ cells. J Exp Med 1994;180:353-8.

40.Scheller L.F., Green S.J., Azad A.F. Inhibition of nitric oxide interrupts the accumulation of CD8+ T cells surrounding Plasmodium berghei-infected hepatocytes. Infect Immun 1997;65:3882-8.

41.Lyons C.R., Orloff G.J., Cunningham J.M. Molecular cloning and functional expression of an inducible nitric oxide synthase from a murine macrophage cell line. J Biol Chem 1992;267:6370-4.

42.Xie Q.W., Cho H.J., Calaycay J., et al. Cloning and characterization of inducible nitric oxide synthase from mouse macrophages. Science 1992;256:225-8.

43.Lancaster J.R.J. A tutorial on the diffusibility and reactivity of free nitric oxide. Nitric Oxide 1997;1:18-30.

44.Ghigo D., Todde R., Ginsburg H., et al. Erythrocyte stages of Plasmodium falciparum exhibit a high nitric oxide synthase (NOS) activity and release an NOS-inducing soluble factor. J Exp Med 1995;182:677-88.

45.Pawloski J.R., Swaminathan R.V., Stamler J.S. Cell-free and erythrocytic S-nitrosohemoglobin inhibits human platelet aggregation. Circulation. 1998;97:263-267.

46.Rockett K.A., Awburn M.M., Cowden W.B., et al. Killing of Plasmodium falciparum in vitro by nitric oxide derivatives. Infect Immun 1991;59:3280-3.

47.Venturini G., Colasanti M., Salvati L., et al. Nitric oxide inhibits falcipain, the Plasmodium falciparum trophozoite cysteine protease. Biochem Biophys Res Commun 2000;7(267):190-3.

48.Taylor-Robinson A.W., Looker, M. Sensitivity of malaria parasites to nitric oxide at low oxygen tensions. Lancet 1998;30(351):1630.

49.Prada J., Malinowski J., Muller S., et al. Effects of Plasmodium vinckei hemozoin on the production of oxygen radicals and nitrogen oxides in murine macrophages. Am J Trop Med Hyg 1996;54:620-4

50.Paul A., Bryant C., Lawson M.F., et al. Dissociation of lipopolysaccharide-mediated induction of nitric oxide synthase and inhibition of DNA synthesis in RAW 264.7 macrophages and rat aortic smooth muscle cells. $\mathrm{Br} \mathrm{J}$ Pharmacol 1997;120:1439-44.

51.Shultz P.J., Archer S.L., Rosenberg M.E. Inducible nitric oxide synthase mRNA and activity in glomerular mesangial cells. Kidney Int 1994;46:683-9.

52.Vyas P., Attur M., Ou G.M., et al. Thin layer chromatography: an effective method to monitor citrulline synthesis by NOS activity. In: Moncada S., Stamler J., Gross S., et al. eds. The biology of nitric oxide, London, Portland Press Ltd., 1996.

53.Xie Q.W., Whisnant R., Nathan C. Promoter of the mouse gene encoding calcium-independent nitric oxide synthase confers inducibility by interferon gamma and bacterial lipopolysaccharide. J Exp Med 1993;177:1779-84.
54.Nussler .K., Di Silvio M., Billiar T.R., et al. Stimulation of the nitric oxide synthase pathway in human hepatocytes by cytokines and endotoxin. J Exp Med 1992;176:261-4.

55.Clark I.A. Does endotoxin cause both the disease and parasite death in acute malaria and babesiosis? Lancet 1978;2:75-7.

56.Hom G.J., Grant S.K., Wolfe G., et al. Role of inducible nitric oxide synthase in septic shock-induced hypotension and vaccular hyporeactivity in rat: tissue analysis of nitric oxide synthase mRNA \& protein expression in the presence \& absence of dexamethasone, $\mathrm{N}^{\mathrm{G}}$-MMLA or indomethacin. In: Moncada S., Stamler J., Gross S., et al. eds. Biology of nitric oxide, London, UK, Portland Press, 1996.

57.Liu S., Adcock I.M., Old R.W., et al. Lipopolysaccharide treatment in vivo induces widespread tissue expression of inducible nitric oxide synthase mRNA. Biochem Biophys Res Commun 1993;96:1208-13.

58.Kasai K., Hattori Y., Banba N., et al. Induction of nitric oxide synthase in human thyrocytes. In: Moncada S., Stamler J., Gross S., et al. eds. Biology of nitric oxide, London, UK, Portland Press, 1996.

59.Mizutani A., Maki H., Torii Y., et al. Ascorbate-dependent enhancement of nitric oxide formation in activated macrophages. Nitric Oxide 1998;2:235-41.

60.Ogura T., Kurashima Y., Esumi H. Regulation of nitric oxide synthase isoform gene expression in human neuronal cells. In: Moncada S., Stamler J., Gross S., et al. eds. Biology of nitric oxide, London, UK, Portland Press, 1996.

61.Nahrevanian H., Dascombe M.J. Nitric oxide and reactive nitrogen intermediates in lethal and nonlethal strains of murine malaria. Parasite Immunol 2001;23:491-501.

62.Nahrevanian H., Dascombe M.J. Expression of inducible nitric oxide synthase (iNOS) mRNA in target organs of lethal and non-lethal strains of murine malaria, Parasite Immunology 2002;24(9-10):471-8.

63.Favre N., Ryffel B., Rudin W. The development of murine cerebral malaria does not require nitric oxide production. Parasitol 1999;118:135-8.

64.Favre N., Ryffel B., Rudin W. Parasite killing in murine malaria does not require nitric oxide production. Parasitol 1999;118:139-43.

65.Granger D.L., Taintor R.R., Boockvar K.S., et al. Measurement of nitrate and nitrite in biological samples using nitrate reductase and Griess reaction. In: Moncada S., Stamler J., Gross S., et al. eds. Biology of nitric oxide, London, UK, Portland Press, 1996.

66.Mohan K., Stevenson M.M. Acquired immunity to asexual blood stages. In: Sherman I.W. ed. Malaria, Parasite biology, Pathogenesis and Protection, Washington D.C., USA, 1st edition, ASM Press, American Society for Microbiology, 2000.

67.Coleman R.M., Rencricca N.J., Stout J.P., et al. Splenic mediated erythrocyte cytotoxicity in malaria. Immunol 1975;29:49-54.

68.Quinn T.C., Wyler D.J. Resolution of acute malaria (Plasmodium berghei in the rat): reversibility and spleen dependence. Am J Trop Med Hyg 1980;29:1-4.

69.Oster C.N., Koontz L.C., Wyler D.J. Malaria in asplenic mice: effects of splenectomy, congenital asplenia and splenic reconstitution on the course of infection. Am J Trop Med Hyg 1980;29:1138-42. 
70.Yoneto T., Yoshimoto T., Wang C.R., et al. Gamma interferon production is critical for protective immunity to infection with blood-stage Plasmodium berghei XAT but neither NO production nor NK cell activation is critical. Infect Immun 1999;67:2349-56.

71.Wyler D.J. Splenic functions in malaria. Lymphology 1983;16:121-7.

72.Dockrell H.M., De Souza J.B., Playfair J.H. The role of the liver in immunity to blood-stage murine malaria. Immunol 1980;41:421-30.

73.Stevenson M.M., Kraal G. Histological changes in the spleen and liver of C57BL/6 and A/J mice during Plasmodium chabaudi AS infection. Exp Mol Pathol 1989;51:80-95.

74.Sayles P.C., Cooley A.J., Wassom D.L. A spleen is not necessary to resolve infections with Plasmodium yoelii. Am J Trop Med Hyg 1991;44:42-8.

75.Brinkmann V., Kaufmann S.H., Simon M.M., et al. Role of macrophages in malaria: $\mathrm{O}_{2}$ metabolite production and phagocytosis by splenic macrophages during lethal Plasmodium berghei and self-limiting Plasmodium yoelii infection in mice. Infect Immun 1984;44:743-6.

76.Gross A., Geva S., Frankenburg S. Plasmodium berghei: lymphocyte and macrophage dynamics in the spleen of Balb/ $c$ mice in the course of infection and after rechallenge of cured mice. Exp Parasitol 1988;65:50-60.

77.Wyler D.J. The spleen in malaria. Ciba Found Symp 1983;94:98-116.

78.Langhorne J., Gillard S., Simon B., et al. Frequencies of CD4+ T cells reactive with Plasmodium chabaudi chabaudi: distinct response kinetics for cells with Th1 and Th2 characteristics during infection. Int Immunol 1989;1:416-24.

79.Allison A.C., Eugui E.M. The role of cell-mediated immune responses in resistance to malaria, with special reference to oxidant stress. Annu Rev Immunol 1983;1:361-92.

80.Bordmann G., Favre N., Rudin W. Malaria toxins: effects on murine spleen and bone marrow cell proliferation and cytokine production in vitro. Parasitol 1997;115:475-83.

81.Playfair J.H., de Souza J.B. Lymphocyte traffic and lymphocyte destruction in murine malaria. Immunol 1982;46:125-33.

82.Suss G., Eichmann K., Kury E., et al. Roles of CD4- and CD8bearing $\mathrm{T}$ lymphocytes in the immune response to the erythrocytic stages of Plasmodium chabaudi. Infect Immun 1988;56:3081-8.

83.Fell A.H., Smith N.C. Immunity to asexual blood stages of Plasmodium: Is resistance to acute malaria adaptive or innate? Parasitol Today 1998;14:364-9.

84.Langhorne J., Pells S., Eichmann K. Phenotypic characterization of splenic T cells from mice infected with Plasmodium chabaudi chabaudi. Scand J Immunol 1993;38:521-8.

85.Weidanz W.P., Melancon-Kaplan J., Cavacini L.A. Cellmediated immunity to the asexual blood stages of malarial parasites: animal models. Immunol Lett 1990;25:87-95.

86.Van der Heyde H., Elloso M.M., Roopenian D.C., et al. Expansion of the CD4-, CD8- gamma delta T cell subset in the spleens of mice during non-lethal blood-stage malaria. Eur J Immunol 1993;23:1846-50.

87.Mauel J. Macrophages as host cells and reactive defence cells: Immune reactions to parasites. New York, Gustav Fischer Verlag Stuttgart.Fortscritte der Zoologie, Band 27. Zb1. Bakt. Suppl. 12, 1982.
88.Doherty T.M. T-cell regulation of macrophage function. Curr Opin Immunol 1995; 7:400-4.

89.Taylor-Robinson A.W. Immunity to asexual blood stages of Plasmodia: is resistance to acute malaria adaptive or innate? Parasitol Today 1999;15:208.

90.Carter R., Walliker D. New observations on the malaria parasites of rodents of the Central African Republic - Plasmodium vinckei petteri subsp. nov. and Plasmodium chabaudi, Landau, 1965. Ann Trop Med Parasitol 1975;69:187-96.

91.Cox F.E.G. Major animal models in malaria research: rodent. In: Wernsdorfer W.H., McGregor S.J. eds. Malaria: Principles and Practice of Malariology, UK, Churchill Livingstone, 1988.

92.Landau I., Chabaud A.G. Natural infection by 2 plasmodia of the rodent Thamnomys rutilans in the Central African Republic. C R Acad Sci Hebd Seances 1965;261:230-2.

93.Taylor B.S., Kim Y.M., Wang Q., et al. Nitric oxide downregulates hepatocyte-inducible nitric oxide synthase gene expression. Arch Surg 1997;132:1177-83.

94.Amante F.H., Good M.F. Prolonged Th1-like response generated by a Plasmodium yoelii-specific T cell clone allows complete clearance of infection in reconstituted mice. Parasite Immunol 1997;19:111-26.

95.Hirunpetcharat C., Finkelman F., Clark I.A., Good M.F. Malaria parasite-specific Th1-like $\mathrm{T}$ cells simultaneously reduce parasitemia and promote disease. Parasite Immunol 1999;21:319-29.

96.Rockett K.A., Awburn M.M., Rockett E.J., et al. Possible role of nitric oxide in malarial immunosuppression. Parasite Immunol 1994; 16:243-9.

97.Stevenson M.M., Tam M.F., Wolf S.F., et al. IL-12-induced protection against blood-stage Plasmodium chabaudi AS requires IFN-gamma and TNF-alpha and occurs via a nitric oxide- dependent mechanism. J Immunol 1995;155:2545-56.

98.Taylor-Robinson A.W. Murine models of cerebral malaria: A qualified defence. Parasitol. Today 1995;11:407-8.

99.Basir R., Dascombe M.J. Nitric oxide involvement in rodent malaria. Asia Pacific J. Pharmacol 2000;14:S72-3.

100. Balmer P., Phillips H.M., Maestre A.E., et al. The effect of nitric oxide on the growth of Plasmodium falciparum, $P$. chabaudi and $P$. berghei in vitro. Parasite Immunol 2000;22:97-106.

101. Dascombe M.J., Nahrevanian H. Pharmacological assessment of the role of nitric oxide in mice infected with lethal and nonlethal species of malaria, Parasite Immunol 2003;25(3):149-59.

102. Burdon M.G., Butler A.R., Renton L.M. A study of antibacterial activity of nitric oxide donor compounds. In: Moncada S., Stamler J., Gross S., et al. eds. The biology of nitric oxide, London, Portland Press Ltd., 1996.

103. Helmby H., Troye-Blomberg M. Differential immunoglobulin $\mathrm{E}$ and cytokine responses in $\mathrm{BALB} / \mathrm{C}$ and $\mathrm{C} 57 \mathrm{Bl} / 6$ mice during repeated infections with blood-stage Plasmodium chabaudi malaria. Parasite Immunol 2000;22:185-90.

104. Taylor-Robinson A.W., Stephen Phillips R., Severn A., et al. The role of TH1 and TH2 cells in a rodent malaria infection. Science 1993;260:1931-4.

105. Favila-Castillo L., Monroy-Ostria A., Garcia-Tapia D. Plasmodium chabaudi chabaudi: effect of low parasitemias on immunity in CB6F1 mice. Exp Parasitol 1999;92:73-80. 
106. Jakobsen P.H., Bate C.A., Taverne J., et al. Malaria: toxins, cytokines and disease. Parasite Immunol 1995;17:223-31.

107. Yoshimoto T., Takahama Y., Wang C.R., et al. A pathogenic role of IL-12 in blood-stage murine malaria lethal strain Plasmodium berghei NK65 infection. J Immunol 1998;160:5500-5.

108. Jacobs P., Radzioch D., Stevenson M.M. In vivo regulation of nitric oxide production by tumor necrosis factor alpha and gamma interferon, but not by interleukin-4, during blood stage malaria in mice. Infect Immun 1996;64:44-9.

109. Abbas A.K., Lichtman A.H., Pober J.S. Effector mechanisms of immune responses. In: Abbas A.K., Lichtman A.H., Pober J.S. eds. Cellular and Molecular Immunology, USA, W.B. Saunders Company, 3rd edition, 1997.

110. Geller D.A., Nussler A.K., Di Silvio M., et al. Cytokines, endotoxin, and glucocorticoids regulate the expression of inducible nitric oxide synthase in hepatocytes. Proc Natl Acad Sci 1993;90:522-6.

111. Grau G., Piguet P.F., Pointaire P. Cytokines and Malaria; Duality of effects in pathology and protection. In: Kunkel S.L., Remick D.G. eds. Cytokines in health and disease, USA, New York, Marcel Dekker, Inc., 1992.
112. Bogdan C., Rollinghoff M., Diefenbach A. The role of nitric oxide in innate immunity. Immunol Rev 2000;173:17-26.

113. Stefano G.B., Magazine H.I. Nitric oxide autoregulation and its significance. Modern Aspects Immunobiol 2001;1:182-6.

114. Mosmann T.R., Sad S. The expanding universe of T-cell subsets: Th1, Th2 and more. Immunol Today 1996;17:138-46.

115. Liew F.Y. Regulation of nitric oxide synthase in macrophages. In: Moncada S., Stamler J., Gross S., et al. eds. The biology of nitric oxide: 2 Enzymology, Biochemistry and Immunology, London, UK, Portland Press, 1992.

116. Levesque M.C., Hobbs M.R., Anstey N.M., et al. Nitric oxide synthase type 2 promoter polymorphisms, nitric oxide production, and disease severity in Tanzanian children with malaria. J Infect Dis 1999;180:1994-2002.

117. Brown G.V., Beck H., Molyneux M., et al. Molecular approaches to epidemiology and clinical aspects of malaria. Parasitol Today 2000;16:448-51.

118. Kun J.F., Mordmuller B., Lell B., et al. Polymorphism in promoter region of inducible nitric oxide synthase gene and protection against malaria. Lancet 1998;351:265-6. 